LYVE1 is differentially expressed in the lymph node and brain metastases of patients with metastatic breast cancer.

Shahan Mamoor, MS 1

1shahanmamoor@gmail.com

East Islip, NY USA

Metastasis to the brain is a clinical problem in patients with breast cancer ${ }^{1-3}$. We mined published microarray data 4,5 to compare primary and metastatic tumor transcriptomes to discover genes associated with brain metastasis in patients with metastatic breast cancer. We found that lymphatic vessel endothelial hyaluronan receptor 1, encoded by LYVE1, was among the genes whose expression was most different in the brain and lymph node metastases of patients with metastatic breast cancer as compared to normal breast tissues. LYVE1 mRNA was present at significantly reduced quantities in brain metastatic tissues as compared to normal breast tissues. These data combined suggest some level of common origin for metastases that reside in the lymph nodes and colonize the brain.

Keywords: breast cancer, metastasis, brain metastases, central nervous system metastases, lymph node metastasis, LYVE1, systems biology of breast cancer, targeted therapeutics in breast cancer. 
One report described a 34\% incidence of central nervous system metastases in patients treated with trastuzumab for breast cancer ${ }^{2}$. More recently, the NEfERT-T clinical trial which compared administration of either neratinib or trastuzumab in conjunction with paclitaxel demonstrated that in a randomized, controlled setting, in breast cancer patients treated with neratinib, not only was the incidence of central nervous system recurrence significantly lower, the time to central nervous system metastasis was significantly delayed as compared to patients administered trastuzumab. The alarmingly high rate of central nervous system metastasis described, as well as data, both anecdotal ${ }^{2}$ and from a randomized, controlled setting ${ }^{6}$ illustrating that treatment with trastuzumab may be associated with these events demands an enhanced understanding of the transcriptional makeup of brain metastatic tissues to support identification of therapeutic targets, whether they are treatment related or not. We performed a global comparative analysis of primary and metastatic tumors in patients with brain metastatic breast cancer ${ }^{4,5}$. We discovered significant differential and decreased expression of lymphatic vessel endothelial hyaluronan receptor 1, encoded by LYVE1, in brain metastatic tissues of patients with metastatic breast cancer.

We performed global comparative transcriptome analysis of metastatic tumor tissues of patients with metastatic breast cancer using published microarray data ${ }^{4,5}$ to describe the transcriptional landscape of brain and lymph node metastasis in human breast cancer in an unbiased fashion and for the discovery of novel therapeutic targets.

LYVE1 is differentially expressed in the brain metastases of patients with brain metastatic breast cancer.

Identification of differentially expressed genes across datasets in cancer can be difficult and more challenging than when doing so in benign organ tissue and sorted cell populations due to higher levels of heterogeneity in tumors, and differing methods of tumor sampling between laboratories. Thus, we asked how gene expression was most significantly different between brain metastases from patients with breast cancer and normal breast tissue as opposed to primary tumors of the breast, by comparing global gene expression profiles of 10 normal breast tissues to 35 brain metastases ${ }^{4}$. Using this strategy, we identified the lymphatic vessel endothelial hyaluronan receptor 1 LYVE1, as among the genes whose expression was most significantly different in the brain metastases of patients with brain metastatic breast cancer 4 (Table 1). When sorting each of the genes expressed in brain metastases based on significance of 
difference in expression between brain metastases and normal breast tissues, LYVE1 ranked 46 out of 41093 total transcripts (Table 1), equating to $99.9 \%$ differential expression. Differential expression of LYVE1 in brain metastases from patients with brain metastatic breast cancer in this dataset was statistically significant $(p=2.42 \mathrm{E}-14)$.

\section{LYVE1 is differentially expressed in the lymph node metastases of patients with metastatic breast cancer.}

Next, we queried a second microarray dataset ${ }^{5}$ generated using lymph node metastases and normal breast tissues from patients with breast cancer to determine whether we could validate differential expression of LYVE1 in metastatic tissues of humans with breast cancer, and whether differential expression of LYVE1 was specific to brain metastases or could be observed in metastases to other sites in patients with metastatic breast cancer. We observed significant differential expression of LYVE1 in lymph node metastases of patients with metastatic breast cancer. When sorting each of the genes expressed in lymph node metastases based on significance of difference as compared to normal breast tissues in patients with breast cancer, LYVE1 ranked 162 out of 22283 total transcripts (Table 2), equating to $66.1 \%$ differential expression. Differential expression of LYVE1 in the lymph node metastases of patients with metastatic breast cancer was statistically significant (Table $2 ; p=1.27 \mathrm{E}-12$ ). Thus, differential expression of LYVE1 was conserved in the brain and lymph node metastases of patients with metastatic breast cancer in two independent microarray datasets ${ }^{4,5}$.

\section{LYVE1 is expressed at significantly lower levels in the brain metastases of patients with brain metastatic breast cancer.}

We obtained exact mRNA expression levels for LYVE1, in normal breast tissues and in brain metastasis of patients with brain metastatic breast cancer to determine direction and statistical significance of change in LYVE1 expression in brain metastatic tissues. LYVE1 was expressed at lower levels in the brain metastases of patients with breast cancer as compared to normal breast tissues, and this difference was statistically significant (Figure $1 ; p<0.0001$ ).

Thus, by mining published microarray data 4,5 in an unbiased and systematic fashion, we identified the lymphatic vessel endothelial hyaluronan receptor 1 LYVE1 as among the genes whose expression was most different, transcriptome-wide, in the brain and lymph node metastases of patients with breast cancer when compared to the normal breast; LYVE1 expression, as compared to the benign breast, was significantly lower in metastasis to the brain.

\section{Discussion}

We provided evidence here that the lymphatic vessel endothelial hyaluronan receptor 1 , encoded by LYVE1, is among the genes whose expression is most different in the brain and lymph node metastases of patients with brain metastatic brain cancer. Evaluation of the effects of ectopic expression of LYVE1 in mouse models of metastatic breast cancer on metastasis to the central nervous system are merited. Differential expression of lymphatic vessel endothelial hyaluronan receptor 1 in metastasis to the lymph nodes and to the brain suggests some level of common origin for metastases that reside in the lymph nodes, successfully evade immune clearance, and subsequently colonize the brain. 


\section{References}

1. Lin, N.U., Amiri-Kordestani, L., Palmieri, D., Liewehr, D.J. and Steeg, P.S., 2013. CNS metastases in breast cancer: old challenge, new frontiers.

2. Bendell, J.C., Domchek, S.M., Burstein, H.J., Harris, L., Younger, J., Kuter, I., Bunnell, C., Rue, M., Gelman, R. and Winer, E., 2003. Central nervous system metastases in women who receive trastuzumab-based therapy for metastatic breast carcinoma. Cancer, 97(12), pp.2972-2977.

3. Tsukada, Y., Fouad, A., Pickren, J.W. and Lane, W.W., 1983. Central nervous system metastasis from breast carcinoma autopsy study. Cancer, 52(12), pp.2349-2354.

4. Salhia, B., Kiefer, J., Ross, J.T., Metapally, R., Martinez, R.A., Johnson, K.N., DiPerna, D.M., Paquette, K.M., Jung, S., Nasser, S. and Wallstrom, G., 2014. Integrated genomic and epigenomic analysis of breast cancer brain metastasis. PloS one, $9(1)$, p.e85448.

5. Sinn, B.V., Fu, C., Lau, R., Litton, J., Tsai, T.H., Murthy, R., Tam, A., Andreopoulou, E., Gong, Y., Murthy, R. and Gould, R., 2019. SET ER/PR: a robust 18-gene predictor for sensitivity to endocrine therapy for metastatic breast cancer. NPJ breast cancer, 5(1), pp.1-8.

6. Awada, A., Colomer, R., Inoue, K., Bondarenko, I., Badwe, R.A., Demetriou, G., Lee, S.C., Mehta, A.O., Kim, S.B., Bachelot, T. and Goswami, C., 2016. Neratinib plus paclitaxel vs trastuzumab plus paclitaxel in previously untreated metastatic ERBB2-positive breast cancer: the NEfERT-T randomized clinical trial. JAMA oncology, 2(12), pp.1557-1564. 
Table 1: LYVE1 is differentially expressed in brain metastases in patients with metastatic breast cancer when compared to normal breast tissues.

Rank of differential expression, probe ID, $p$-value with respect to differential expression, $t$, a moderated tstatistic, B, the log-odds of differential expression between the two groups compared, gene and gene name are listed in this chart.

PAGE 5 
Table 2: LYVE1 is differentially expressed in lymph node metastases of patients with metastatic breast cancer when compared to normal breast tissue.

Rank of differential expression, probe ID, $p$-value with respect to differential expression, $\mathrm{t}$, a moderated tstatistic, B, the log-odds of differential expression between the two groups compared, gene and gene name are listed in this chart. 


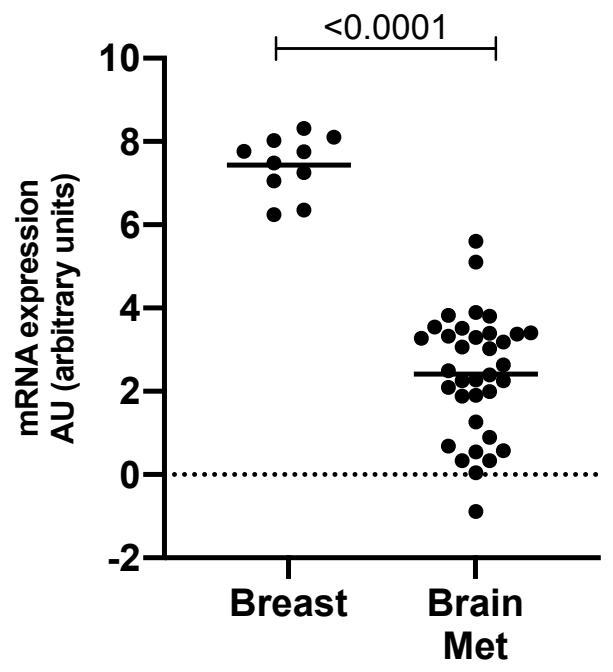

Figure 1: LYVE1 is expressed at significantly lower levels in the brain metastases of patients with brain metastatic breast cancer as compared to normal breast tissues.

The mRNA expression level of LYVE1 in normal breast tissues (left) and in the brain metastases of brain metastatic breast cancer patients (right) is graphically represented here with mean LYVE1 levels marked and the result of a statistical test evaluating difference in mRNA expression level between brain metastases and normal tissues of the breast, a $p$-value, listed above. 